\title{
A Comparison of the Polyphenolic and Free Radical Scavenging Activity of Cold Brew versus Hot Brew Black Tea (Camellia Sinensis, Theaceae)
}

\author{
Chathuranga Manhari Magammana ${ }^{1}$, Cheryl Rosita Rock $^{1}$, Long Wang $^{1}$ \& Virginia Gray ${ }^{1}$ \\ ${ }^{1}$ Department of Family and Consumer Sciences, California State University, Long Beach, 90840, USA \\ Correspondence: Dr. Cheryl Rock, 1250 Bellflower Boulevard, Long Beach, CA 90840, USA. Tel: \\ 562-985-4497. E-mail: cheryl.rock@csulb.edu
}

Received: October 28, 2019

Accepted: November 26, 2019 Online Published: April 2, 2019

doi:10.5539/jfr.v8n3p35

\author{
URL: https://doi.org/10.5539/jfr.v8n3p35
}

\begin{abstract}
Recently, a new trend called cold brewing gained popularity in the tea and coffee beverage industry. Cold brew and hot brew black tea may have different sensory qualities and antioxidant levels because of their polyphenolic properties and brewing processes. The objectives of this study were to determine antioxidant properties and polyphenolic content of commercial brands of cold brew and hot brew black tea. The total phenolic content of the cold brew tea was determined to be $0.19 \mathrm{mg} / \mathrm{mL}$ gallic acid equivalents $/ 100 \mathrm{~g}$ and hot brew tea was 0.43 $\mathrm{mg} / \mathrm{mL}$ gallic acid equivalents $/ 100 \mathrm{~g}$ when assayed by Folin-Ciocalteu's reagent method. The total flavonoid content of the cold brew tea was $0.40 \mathrm{mg} / \mathrm{mL}$ catechin equivalents $/ 100 \mathrm{~g}$ and hot brew was $1.01 \mathrm{mg} / \mathrm{mL}$ catechin equivalents/100 g. Moreover, antioxidant capacity of cold brew and hot brew black tea was analyzed where their ability to scavenge DPPH radicals was $86.3 \%$ and $88.1 \%$ respectively. There was a significant difference in total phenolic content between hot brew and cold brew $(p=0.004)$. Similarly, there was a significant difference in total flavonoid between cold brew and hot brew $(p=0.004)$. Additionally, there was a significant difference in DPPH scavenging activity between cold brew and hot brew $(p=0.016)$. Overall, it can be concluded that although cold brew tea contained a lower amount of phenolics and flavonoids as compared to hot brew tea, they both were able to scavenge DPPH radicals in nearly same capacity.
\end{abstract}

Keywords: antioxidants, cold brew, hot brew, tea

\section{Introduction}

\subsection{Consumption Trends and Tea Types}

Teas are classified as the following: black, green, oolong and white tea, which are generally processed using the standard steps: withering, rolling, fermentation (oxidation) and drying (firing) with slight variations based on the type (Shinde, Das, \& Datta, 2013). All tea varieties come from leaves of the Camellia Sinensis (L.) plant (Carloni et al., 2013). However, black tea, the main focus of this research, is a fully fermented type (Shinde et al., 2013). Black tea is the most consumed (80\%; Nie, Dong, Bai, \& Xia, 2014) and produced (78\%; Ruxton \& Mason, 2011) variety in the world (Striegel, Kang, Pilkenton, Rychlik, \& Apostolidis, 2015).

\subsection{Cold Brewing in Tea and Coffee Industry}

Recently, a new process of cold brew has gained popularity in the beverage industry (Refermat, 2017). Until major coffee franchises introduced cold brew coffee in the United States (U.S.) in 2015, it was a small market niche with which only few consumers were familiar (Kline, 2016). Especially in the coffee industry, cold brew is drawing the attention of a new generation referred to as millennials (aged 18-34; Fry, 2016) in that many of them consider it as their drink of choice (Strand, 2017). Importantly, successful outcomes of cold brew method illustrate the trends toward healthier foods (Kline, 20106). With increased consumer demands, new frequent innovations and products such as unique tea products similar to cold brew coffee continue to expand the beverage industry (Tea Association of the U.S.A. Inc., 2017).

\subsection{Innovation of Cold Brew Tea Product}

Cold brew tea products are produced using low temperature $\left(15^{\circ} \mathrm{C}\right)$ or cold water $\left(4{ }^{\circ} \mathrm{C}\right)$ and have the benefits of traditional tea as it relates to established literature on their antioxidant properties (He, Liu, \& Huang, 2011). 
Other specific benefits include the convenience of preparation while enjoying a freshly brewed tea taste (Balentine et al., 2004). Moreover, the cold brew method minimizes the bitterness and astringency of caffeine since less tannins are extracted from the tea leaves as compared to hot brewing (Dobos, 2017). As a consequence, cold brew tea began to spark the interest since it can create smoother and sweet iced tea also (Tea Association of the U.S.A. Inc., 2017).

\subsection{Oxidative Stress and Tea Antioxidants}

Tea is known to contain plant compounds which are believed to prevent chronic diseases (Koutelidakis et al., 2016). Some of these polyphenols in tea include catechins or flavan-3-ols, theaflavins, thearubigins and proanthocyanidins (Chen, Qu, Fu, Dong, \& Zhang, 2009) and have been implicated in preventing cancer, obesity, type 2 diabetes and cardiovascular disease (CVD; Fernando \& Soysa, 2015). Mechanisms include inhibiting lipoprotein oxidation, decreasing oxidative stress (OS) and controlling inflammation biomarkers (Koutelidakis et al., 2016). Polyphenols also have been shown to decrease serum glucose, triglyceride and low-density lipoprotein (LDL)/high-density lipoprotein (HDL) plasma cholesterol ratio and to improve plasma antioxidant level, which may help to lower the risk of CVD (Pan et al., 2013).

\subsection{Importance of Tea Brewing Methods Research}

There is an abundance of research on tea and antioxidants; however, there is a limited research on comparing antioxidant activity in cold versus hot brew black tea; in particular commercially made cold brew black tea. Specifically, in literature, there were some studies that analyzed antioxidant levels in different tea types by using various brewing times and temperatures. However, there were limited studies that tested antioxidants in black tea, which is the most consumed and produced tea type globally. The caveat with these studies is that cold-water tea infusions were prepared by using the teas that are supposed to be brewed with hot water brewing methods. This error can affect test results on antioxidant properties and quality of teas. Therefore, it is important to study the polyphenolic content as well as the antioxidant capacity of the cold brew and hot brew black tea, as they could have different antioxidant levels when utilizing the proper techniques or protocols in their brewing methods. In the same manner, it is important to consider that antioxidant properties of certain teas are best acquired with the inscribed (hot water) conditions. In addition, it is important to study the polyphenolic content and antioxidant power of cold and hot brew black tea as cold brew preparation is receiving a great attention of consumers such as millennials recently and the consumers can be more knowledgeable about amount and quality of tea antioxidants that are available in the products. The overall objective of this study was to compare the polyphenolic content and free radical scavenging activity of cold brew and hot brew black teas.

\section{Method}

\subsection{Tea Brewing}

For both cold brew and hot brew black tea, $2 \mathrm{~g}$ of each tea sample respectively were brewed in $230 \mathrm{~mL}$ of cold (4 $\left.{ }^{\circ} \mathrm{C}\right)$ and hot $\left(100{ }^{\circ} \mathrm{C}\right)$ water for 5 minutes as prescribed on the commercial package.

\subsection{Antioxidant Capacity Determination}

\subsubsection{Determination of the Free radical Scavenging Activity of Black Tea}

The free-radical scavenging activity of the prototype was measured with reference to Priyanka, Kadam, Kadam, Ghule, \& Aparadh (2013). First, $1 \mathrm{~mL}$ of each extract was added to $3 \mathrm{~mL}$ of $0.1 \mathrm{M}$ DPPH (1, 1-Diphenyl-2-picryl-hydrazyl) radical solution. The DPPH radical was dissolved in $90 \%$ methanol, shaken, and incubated at ambient temperature (about $23{ }^{\circ} \mathrm{C} / 73{ }^{\circ} \mathrm{C}$ ) for 30 minutes with minimal light exposure. Then, analysis of DPPH was conducted using a UV Spectrometer (Thermo Scientific AquaMate 8000 UV-Vis Spectrophotometer) at a wavelength of $517 \mathrm{~nm}$. Free radical scavenging activity was calculated as the percentage (\%) of free radicals scavenged by the extracts (Equation 1). Also, the blank was $1 \mathrm{~mL}$ of methanol to $3 \mathrm{~mL}$ of DPPH.

$$
\% \mathrm{DPPH} \text { of Sample }=\mathrm{A}_{\text {blank }}-\mathrm{A}_{\text {sample }} / \mathrm{A}_{\text {blank }} \mathrm{X} 100
$$

\subsection{Polyphenolic Content Determination}

\subsubsection{Phenolic Content of Black Tea}

Total phenolics were measured since they contribute to the antioxidant capacity and potential of cold brew and hot brew black tea products as described by Marinova, Ribarova, \& Atanassova (2005). One $\mathrm{mL}$ of each of the sample extracts as well as a series of gallic acid standard solutions $(0.02,0.04,0.06,0.08$ and $0.10 \mathrm{mg} / \mathrm{mL})$ were added to $25 \mathrm{~mL}$ volumetric flasks, containing $9 \mathrm{~mL}$ of deionized distilled water $\left(\mathrm{DDH}_{2} \mathrm{O}\right)$. Subsequently, $1 \mathrm{~mL}$ of Folin-Ciocalteu's (FC) reagent was added to the mixture and shaken. After 5 minutes, $10 \mathrm{~mL}$ of $7 \%$ sodium 
bicarbonate $\left(\mathrm{Na}_{2} \mathrm{CO}_{3}\right)$ solution was added to the mixture in each flask. The solution in each flask was diluted to volume $(25 \mathrm{~mL})$ using $\mathrm{DDH}_{2} \mathrm{O}$ and was mixed. Following that, the flasks were incubated for 90 minutes at room temperature and the absorbance of the mixtures were read at $750 \mathrm{~nm}$ using an Orion Aquamate $8000 \mathrm{UV}$ spectrometer. Then, the total phenolics of the cold brew and hot brew black tea were expressed as gallic acid equivalents (GAE)/100 $\mathrm{g}$ fresh weight. Last, all samples were analyzed in triplicate.

\subsubsection{Flavonoid Content of Black Tea}

A standard colorimetric assay as described by Marinova et al. (2005) with slight modifications was used to quantify total flavonoid content. First, one $\mathrm{mL}$ of a series of catechin standard solutions $(0.2,0.4,0.6,0.8$ and 1.0 $\mathrm{mg} / \mathrm{mL}$ ) was added to $10 \mathrm{~mL}$ volumetric flasks containing $4 \mathrm{~mL}$ of $\mathrm{DDH}_{2} \mathrm{O}$ respectively. Then, to each flask, 0.3 $\mathrm{mL}$ of $5 \%$ sodium nitrate $\left(\mathrm{NaNO}_{2}\right)$ was added. After 5 minutes, $0.3 \mathrm{~mL}$ of $10 \%$ aluminum chloride $\left(\mathrm{AlCl}_{3}\right)$ was added to each flask, followed by $2 \mathrm{~mL}$ of $1 \mathrm{M}$ sodium hydroxide $(\mathrm{NaOH})$. The mixture in each flask was filled to volume $(10 \mathrm{~mL})$ using $\mathrm{DDH}_{2} \mathrm{O}$ and was mixed well. Using an Orion Aquamate $8000 \mathrm{UV}$ spectrometer, the absorbance of the samples was read wavelength at $510 \mathrm{~nm}$. Finally, the total flavonoid contents were expressed as $\mathrm{mg}$ catechin equivalents (CE)/100 $\mathrm{g}$ of tea. All samples were analyzed in triplicate.

\subsection{Data Analysis}

All numerical data of this study were analyzed by using the International Business Machine (IBM) Statistical Package for the Social Sciences (SPSS), Version 24. Mean and the standard deviation (SD) of the each chemical test were used. For all chemical tests above mentioned, Mann-Whitney tests (non-parametric test) were used to compare the differences in mean phenolic content, flavonoid content and antioxidant capacities of the cold brew versus hot brew black tea. $P<0.05$ was considered to be statistically significant.

\section{Results}

\subsection{Total Phenolic Content}

Figure 1 shows the results of gallic acid standard curve equation $\left(y=3.995 x+0.0137, r^{2}=0.9917\right)$ for total phenolic content in our experiment. In this study, the total phenolic content was lower in cold brew black tea $(0.19 \mathrm{mg} / \mathrm{mL}$ gallic acid equivalents (GAE)/100 $\mathrm{g}$ or $43.7 \mathrm{mg} / 230 \mathrm{~mL}$ GAE$/ 100 \mathrm{~g}$ and mean absorbance value was $0.78 \pm 0.04)$ than hot brew black tea $(0.43 \mathrm{mg} / \mathrm{mL} \mathrm{GAE} / 100 \mathrm{~g}$ or $98.9 \mathrm{mg} / 230 \mathrm{~mL} \mathrm{GAE} / 100 \mathrm{~g}$ and mean absorbance value was $1.74 \pm 0.10$; Table 1 ). Moreover, the findings showed that there was significant difference in total phenolic content between cold brew and hot brew $(p=0.004$; Table 2$)$.

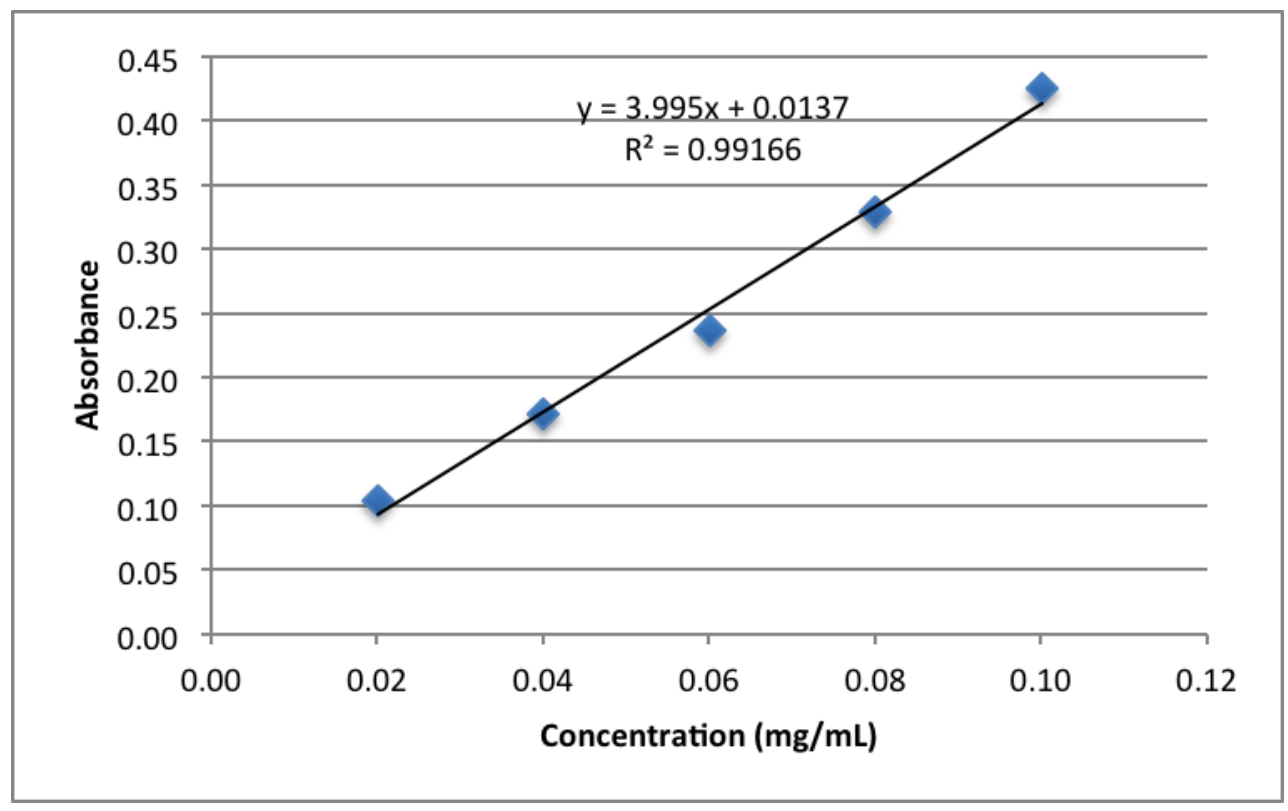

Figure 1. Standard curve for total phenolics content

\subsection{Total Flavonoid Content}

Figure 2 shows the results of catechin standard curve equation $\left(y=0.2465 x+0.0204, r^{2}=0.9787\right)$ for total flavonoid content in our research. In our study, the total flavonoid content was lower in the cold brew tea $(0.40$ 
$\mathrm{mg} / \mathrm{mL}$ catechin (CE) equivalents $/ 100 \mathrm{~g}$ of extract or $92.0 \mathrm{mg} / 230 \mathrm{~mL} \mathrm{CE} / 100 \mathrm{~g}$ and mean absorbance value was $0.12 \pm 0.01)$ than hot brew tea $(1.01 \mathrm{mg} / \mathrm{mL} \mathrm{CE} / 100 \mathrm{~g}$ or $232 \mathrm{mg} / 230 \mathrm{~mL} \mathrm{CE} / 100 \mathrm{~g}$ and mean absorbance value was $0.27 \pm 0.02$; Table 1). Additionally, the results showed that there was a significant difference in total flavonoid between cold brew and hot brew $(p=0.004$; Table 2).

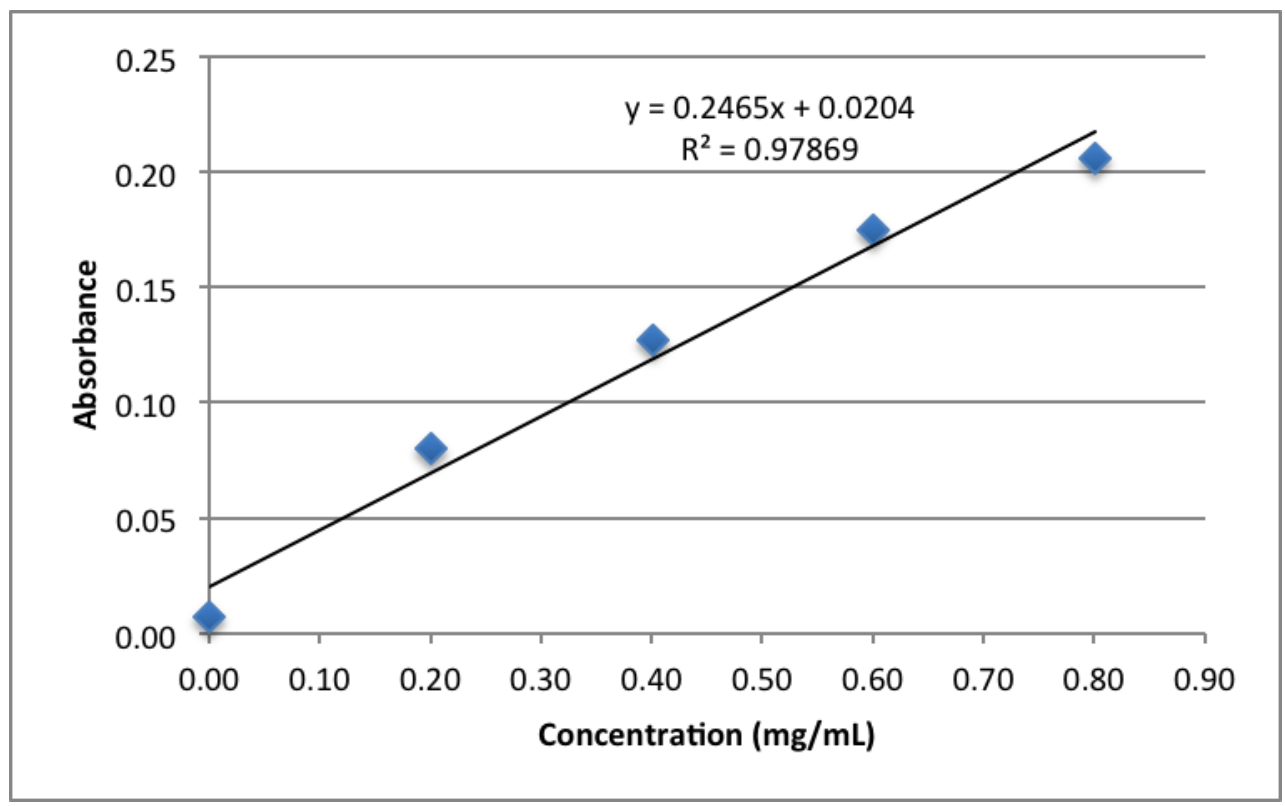

Figure 2. Standard curve for total flavonoids content

\subsection{Determination of the Free Radical Scavenging Activity of Tea}

In addition, in this study, antioxidant capacity of cold brew and hot brew black tea was analyzed by DPPH chemical assay. To illustrate, the ability of cold brew and hot brew black tea to scavenge DPPH radical was $86.3 \%$ (mean absorbance value was $0.61 \pm 0.06$ ) and $88.1 \%$ (mean absorbance value was $0.53 \pm 0.01$ ) respectively (Table 1). The high percentages show high power of fighting off of free radicals by the tea antioxidants. Moreover, the results indicated that there was a significant difference in DPPH scavenging activity between cold brew tea and hot brew tea $(p=0.016$; Table 2).

Table 1. Polyphenolic content results of cold versus hot brew black tea

\begin{tabular}{llll}
\hline Tea brew type & $\begin{array}{l}\text { Total phenolic content } \\
\text { (mg/mL gallic acid } \\
\text { equivalents }(\mathrm{GAE}) / 100 \mathrm{~g} \\
\text { or } \mathrm{mg} / 230 \mathrm{~mL} \mathrm{GAE} / 100 \mathrm{~g})\end{array}$ & $\begin{array}{l}\text { Total flavonoid content } \\
(\mathrm{mg} / \mathrm{mL} \text { catechin }(\mathrm{CE}) \\
\text { equivalents } / 100 \mathrm{~g} \text { of extract or } \\
\mathrm{mg} / 230 \mathrm{~mL} \mathrm{CE} / 100 \mathrm{~g})\end{array}$ & $\begin{array}{l}\text { DPPH: Free radical } \\
\text { scavenging activity }(\%)\end{array}$ \\
\hline Cold Brew Tea & 0.19 & 0.40 & 86.3 \\
& OR & OR & \\
Hot Brew Tea & 43.7 & 92.0 & 88.1 \\
& 0.43 & 1.01 & \\
& OR & OR & \\
& 98.9 & 232 & \\
\hline
\end{tabular}

Table 2. Mann- Whitney U- test results comparing polyphenolic properties of cold versus hot brew black tea

\begin{tabular}{llll}
\hline Antioxidant test & Cold brew mean \pm SD & Hot brew mean \pm SD & $P$-value \\
\hline Phenolic Test & $0.78 \pm 0.04$ & $1.74 \pm 0.10$ & 0.004 \\
Flavonoid Test & $0.12 \pm 0.01$ & $0.27 \pm 0.02$ & 0.004 \\
DPPH Radical & $0.61 \pm 0.06$ & $0.53 \pm 0.01$ & 0.016 \\
\hline
\end{tabular}




\section{Discussion}

\subsection{Total Phenolic Content}

In our study, cold brew tea had lower phenolic content and may be due to the fact that cold water extracted less phenolics from tea leaves than hot water. Another reason for more efficient extraction of tea phenolics by hot brew could be because tea polyphenols (phenolics) are more water soluble in hot water than cold water. This also showed that cold brew may need more time to extract more phenolics from tea leaves at that temperature. A study (Lantano, Rinaldi, Cavazza, Barbanti, \& Corradini, 2015) investigated total phenol content of tea extraction made with hot water ( $12 \mathrm{~g}$ tea in $1 \mathrm{~L}$ of water of specific temperature), cold tea (tea in $4{ }^{\circ} \mathrm{C}$ water \pm 1 for 12 hours) and ice tea (tea in $80{ }^{\circ} \mathrm{C}$ water and adding ice after required time of infusion and removing tea leaves). The researchers (Lantano et al., 2015) found that the total phenol content (mean \pm SD expressed as $\mathrm{mg} / \mathrm{g}$ leaves) for hot black tea was $14.2 \pm 0.1$, for cold tea was $20.4 \pm 0.1$ and for ice tea was $14.8 \pm 0.2$ (Lantano et al., 2015). However, the results of the study performed by Lantano et al. (2015) were not similar to ours as it was revealed that cold brew tea had higher phenolic content than hot brew tea. This could be due to the fact that they prepared the cold-water tea infusions after brewing the tea in $80^{\circ} \mathrm{C}$ water. Therefore, brewing conditions such as time and temperature could affect the total phenolic content of tea infusions. Another reason could be that we used a commercially available cold brew black tea product in our study.

In addition, another study by Carloni et al. (2013) determined that total phenols of hot brew $\left(90{ }^{\circ} \mathrm{C}\right.$ for 7 minutes) was $(10.7 \pm 4.0 \mathrm{mM} \mathrm{GAE})$ in black crush-tear-curl (CTC) tea and $(14.9 \pm 5.9 \mathrm{mM} \mathrm{GAE})$ in black orthodox tea respectively. This study found a lower amount of total phenols than in the present study and it could be because of use of different black tea brands that were from various parts of the world and use of variety of brewing times and temperatures. Moreover, another study (Abbasian et al., 2013) investigated the total polyphenolic compounds of six medicinal plants and ten commercial tea brands and found that the total phenols (mg GAE/L) of a popular commercial brand of black tea sample was $98.05 \pm 11.37$. In comparison, the numerical results of the total phenols of black tea in their study $(98.05 \pm 11.37 \mathrm{mg}$ GAE$/ \mathrm{L})$ were comparable with our study results $(0.43 \mathrm{mg} / \mathrm{mL} \mathrm{GAE} / 100 \mathrm{~g}$ or $98.9 \mathrm{mg} / 230 \mathrm{~mL} \mathrm{GAE} / 100 \mathrm{~g})$ for hot brew black tea. This could be because of use of same tea brand with the similar tea quality standards to make hot brew tea in both studies.

\subsection{Total Flavonoid Content}

Our results showed that cold brew tea had lower flavonoids content than hot brew tea and it may be due to the fact that extraction of tea flavonoids from tea leaves was more efficient and rapid with hot brew than cold brew technique. This could be due to the fact that solubility of tea polyphenols (flavonoids) in hot water was higher than in cold water. Moreover, it also suggests that cold brewing method may need longer infusion time to extract more tea flavonoids from tea leaves. On the other hand, a study found that total flavonoid content for black orthodox tea was $6.60 \pm 1.52 \mathrm{mM} \mathrm{CE}$ (Carloni et al., 2013). This study had different results than our study and it could be because of use of black tea that was made from different processing methods such as orthodox versus CTC and use of different brewing conditions (different brewing time and temperature and weight of tea used). Furthermore, a study (Abbasian et al., 2013) investigated the total flavonoid content of six medicinal plants and ten commercial tea brands. This study (Abbasian et al., 2013) found that a popular commercial tea brand had the highest total flavonoid content when compared to other tea brand samples. To elaborate, it was observed that the total flavonoid content $(\mathrm{mg} \mathrm{CE} / \mathrm{L})$ of this popular commercial brand of black tea sample was $230.72 \pm 15.20$. According to these results, the total flavonoid content of this specific brand of black tea used in their study was very similar results of our study for hot brew black tea. This could be due to the use of similar brewing times, techniques and temperatures to prepare the teas and use of teas from similar parts of the world to produce the black tea type.

\subsection{Determination of the Free Radical Scavenging Activity of Tea}

Experimentally, we found that cold brew and hot brew black tea both were able to scavenge DPPH radicals in very similar capacity even though there were lower total phenolic and flavonoid content in cold brew tea than hot brew tea. In other words, our results revealed that cold brew tea and hot brew tea were almost effective in same extent in antioxidant activity. Bhuyan et al. (2013) analyzed a total of 60 CTC black tea samples from six regions from India. According to Bhuyan et al. (2013), the mean value of the DPPH scavenging activity was $86.73 \%$ for Barak Valley, $86.37 \%$ for Terai region, $77.57 \%$ for Dooars region, $82.68 \%$ for Upper Assam region, $80.95 \%$ for North Bank region and $80.92 \%$ for South Bank region. As a result, this study's (Bhuyan et al., 2013) results for DPPH assay of black tea samples matched our results. This could be possibly because of use of same tea type in the both studies. 


\subsection{Importance of Findings}

In current literature, there are many studies on tea and antioxidants; however, there are very few studies comparing antioxidants in hot brew black tea versus cold brew black tea, especially commercially made cold brew black tea. In our study, even though cold brew tea had lower phenolics and flavonoids compared to hot brew tea, both cold and hot brew teas were able to scavenge DPPH radical at the same capacity. This could show that there is a certain bioavailability of tea antioxidants (phenolics and flavonoids) in tea regardless of the amount of antioxidants in tea drink. A limitation of our study was that there might be slight differences in the antioxidant properties of cold and hot brew teas since they might have been originally acquired from various tea suppliers to formulate the products. Additionally, the results do not reflect the bioavailability of the antioxidants in a biological system (i.e. in vivo). The repositioning of tea consumption as cold brew tea as an alternative to the traditional hot brew tea makes it necessary and useful for the consumers to know more about the amount and quality of the tea antioxidants that they can acquire by consuming cold brew versus hot brew tea for achieving desired health benefits. Overall, according to the results of our study, it can be concluded that cold brew tea had lower phenolics and flavonoids as compared to hot brew tea. However, both cold and hot brew teas were able to scavenge DPPH radicals at nearly the same capacity. In the food industry, the cold brew tea method can be used to make ready-to-drink cold brew tea in cans and bottles, instant tea and coffee powders that are high in polyphenols and have high antioxidant capacity. As a result, consumers can enjoy tea and coffee instantly and conveniently by using low temperature or cold water with similar antioxidative benefits.

\section{Acknowledgements}

Dr. Cheryl Rock and Chathuranga M. Magammana designed the study, collected the data, interpreted the results, wrote and edited the manuscript. Dr. Long Wang and Dr. Virginia Gray assisted in drafting the manuscript.

\section{References}

Abbasian, S., Karimi, F., Moghaddam, G., Soroush, A., Moloudian, H., \& Ahosseini, M.S. (2013). Antioxidant properties of different black tea samples and some Iranian native plants. Pharmacie Globale, 4(2), 1-5.

Balentine, D. A., Gobbo, S. A., Hang, R. S., Qi, X., Parry, A. D., \& Goodsall, C. W. (2004). Cold brew tea (U. S. Patent No. 6780454). Retrieved from https://www.google.com/patents/US6780454

Bhuyan, L. P., Sabhapondit, S., Baruah, B. D., Bordoloi, C., Gogoi, R., \& Bhattacharyya, P. (2013). Polyphenolic compounds and antioxidant activity of CTC black tea of North-EastIndia. Food Chemistry, 141(4), 3744-3751. https://doi.org/10.1016/j.foodchem.2013.06.086

Carloni, P., Tiano, L., Padella, L., Bacchetti, T., Customu, C., Kay, A., \& Damiani, E. (2013). Antioxidant activity of white, green and black tea obtained from the same tea cultivar. Food Research International, 53(2), 900-908. https://doi.org/10.1016/j.foodres.2012.07.057

Chen, H., Qu, Z., Fu, L., Dong, P., \& Zhang, X. (2009). Physicochemical properties and antioxidant capacity of 3 polysaccharides from green tea, oolong tea, and black tea. Journal of Food Science, 74(6), C469-C474. https://doi.org/10.1111/j.1750-3841.2009.01231.x

Dobos, E. (2017). Cold brew teas gain ground. Retrieved from http://worldteanews.com/news/cold-brew-teas-gain-ground

Fernando, C. D., \& Soysa, P. (2015). Extraction kinetics of phytochemicals and antioxidant activity during black tea (Camellia sinensis L.) brewing. Nutrition Journal, 14, 74. https://doi.org/10.1186/s12937-015-0060-x

Fry, R. (2016). Millennials overtake baby boomers as America's largest generation. Retrieved from http://www.pewresearch.org/fact-tank/2016/04/25/millennials-overtake-baby-boomers/

He, X., Liu, J., \& Huang, Z. (2011). Preparation of cold brew tea by explosion puffing drying at variable temperature and pressure. Drying Technology, 29(8), 888-895. https://doi.org/10.1080/07373937.2010.547969

Kline, D. B. (2016). How cold brew became America's latest coffee craze. Retrieved from https://www.fool.com/investing/2016/07/18/how-cold-brew-became-americas-latest-coffee-craze.aspx

Koutelidakis, A., Andritsos, N., Kabolis, D., Kapsokefalou, M., Drosinos, E., \& Komaitis, M. (2016). Antioxidant and antimicrobial properties of tea and aromatic plant extracts against bacterial foodborne pathogens: A comparative evaluation. Current Topics in Nutraceutical Research, 14(2), 133-142.

Lantano, C., Rinaldi, M., Cavazza, A., Barbanti, D., \& Corradini, C. (2015). Effects of alternative steeping methods on composition, antioxidant property and colour of green, black and oolong tea infusions. Journal 
of Food Science Technology, 52(12), 8276- 8283. https://doi.org/10.1007/s13197-015-1971-4

Marinova, D., Ribarova, F., \& Atanassova, M. (2005). Total phenolics and total flavonoids in Bulgarian fruits and vegetables. Journal of the University of Chemical Technology and Metallurgy, 40(3), 255-260. Retrieved from http://www.researchgate.net/profile/Maria_Atanassova/publication/258769164_TOTAL_PHENOLICS_AN D_TOTAL_FLAVONOIDS_IN_BULGARIAN_FRUITS_AND_VEGETABLES/links/00463528f0a28e54a 6000000.pdf

Nie, X., Dong, D., Bai, Y., \& Xia, P. (2014). Meta-analysis of black tea consumption and breast cancer risk: Update 2013. Nutrition and Cancer, 66(6), 1009-1014. https://doi.org/10.1080/01635581.2014.936947

Pan, M., Lai, C., Wang, H., Loc, C., Hod, C., \& Li, S. (2013). Black tea in chemo-prevention of cancer and other human diseases. Food Science and Human Diseases, 2(1), 12-21. https://doi.org/10.1016/j.fshw.2013.03.004

Priyanka, C., Kadam, D., Kadam A., Ghule, Y., \& Aparadh, V. (2013). Free radical scavenging (DPPH) and ferric reducing ability (FRAP) of some gymnosperm species. International Journal of Research in Botany, 3(2), 34-36. Retrieved from

http://www.researchgate.net/profile/Vishal_Aparadh/publication/239949381_Free_Radical_Scavenging_(D PPH)_and_Ferric_Reducing_Ability_(FRAP)_of_Some_Gymnosperm_species/links/02e7e51c3fd004c8cb0 00000.pdf

Refermat, E. (2017). 12-hour coffee. Automatic Merchandiser, 56(6), 12.

Ruxton, C. H. S., \& Mason, P. (2011). Is black tea consumption associated witha lower risk of cardiovascular disease and type 2 diabetes? Nutrition Bulletin, 37, 4-15. https://doi.org/10.1111/j.1467-3010.2011.01937.x

Shinde, A., Das, S., \& Datta A. K. (2013). Quality improvement of orthodox and CTC tea and performance enhancement by hybrid hot air-radio frequency (RF) dryer. Journal of Food Engineering, 116(2), 444-449. https://doi.org/10.1016/j.jfoodeng.2012.12.001

Strand, O. (2017). How cold brew changed the coffee business. Retrieved from https://www.nytimes.com/2017/06/05/dining/cold-brew-coffee.html

Striegel, L., Kang, B., Pilkenton, S. R., Rychlik, M., \& Apostolidis, E. (2015). Effect of black tea and black tea pomace polyphenols on a-glucosidase and a-amylase inhibition, relevant to type 2 diabetes prevention. Frontiers in Nutrition, 2, 1-6. https://doi.org/10.3389/fnut.2015.00003

Tea Association of the U.S.A. Inc. (2017). State of the industry. Retrieved from http://www.teausa.org/14654/state-of-the-industry

\section{Copyrights}

Copyright for this article is retained by the author(s), with first publication rights granted to the journal.

This is an open-access article distributed under the terms and conditions of the Creative Commons Attribution license (http://creativecommons.org/licenses/by/4.0/). 